\title{
An in vitro endothelial cell protective effect of secretory leukocyte protease inhibitor against simulated ischaemia/reperfusion injury
}

\author{
NITIRUT NERNPERMPISOOTH ${ }^{1,2}$, EAKKAPOTE PROMPUNT $^{1}$ and SARAWUT KUMPHUNE ${ }^{1,3}$ \\ ${ }^{1}$ Biomedical Research Unit in Cardiovascular Sciences (BRUCS); Departments of ${ }^{2}$ Cardio-Thoracic Technology \\ and ${ }^{3}$ Medical Technology, Faculty of Allied Health Sciences, Naresuan University, Phitsanulok 65000, Thailand
}

Received May 30, 2017; Accepted August 10, 2017

DOI: $10.3892 / e t m .2017 .5272$

\begin{abstract}
Endothelial dysfunction is an essential deleterious modulator of ischaemia/reperfusion (I/R) injury. Secretory leukocyte protease inhibitor (SLPI) has demonstrated myocardial protection in cardiac transplantation; however, the effect of SLPI in endothelial I/R injury remains unexplored. In the present study, the effect of recombinant human SLPI (rhSLPI) treatment against endothelial cells (ECs) subjected to simulated I/R injury and the effect of treatment at different time points were determined. Human umbilical vein ECs (HUVECs) were subjected to normoxic or simulated I/R (SI/R) conditions, and rhSLPI at concentrations of 1, 10, 100 and $1,000 \mathrm{ng} / \mathrm{ml}$ was added to the cells prior to ischaemia, during ischaemia or at the onset of reperfusion. Endothelial injury and cytoskeleton disruption were assessed, and western blot analysis was conducted. The results revealed that rhSLPI treatment at $1,000 \mathrm{ng} / \mathrm{ml}$ significantly increased the HUVEC viability under $s \mathrm{I} / \mathrm{R}$ injury $(\mathrm{P}<0.05)$. In addition, treatment with rhSLPI prior to or during ischaemia markedly attenuated the activity of lactase dehydrogenase compared with that in the sI/R group. In addition, the $\mathrm{H}_{2} \mathrm{O}_{2}$-induced reactive oxygen species production was reduced by $\sim 17 \%$ upon rhSLPI pretreatment. Endothelial cytoskeleton disruption was also preserved by rhSLPI added prior to the reperfusion period. Furthermore, pretreatment with rhSLPI promoted protein kinase B activation, as well as reduced p38 mitogen-activated protein kinase phosphorylation and B-cell lymphoma 2-associated X protein expression in response to $\mathrm{I} / \mathrm{R}$ injury. These findings indicated that rhSLPI possesses antioxidant and antiapoptotic properties against endothelial responses to I/R injury. Therefore,
\end{abstract}

Correspondence to: Professor Sarawut Kumphune, Department of Medical Technology, Faculty of Allied Health Sciences, Naresuan University, 99 Moo 9, Thapho, Meung, Phitsanulok 65000, Thailand E-mail: sarawutk@nu.ac.th

Key words: acute myocardial infarction, ischaemia/reperfusion injury, recombinant human secretory leukocyte protease inhibitor, endothelium, cell death, reactive oxygen species the cytoprotective effect of rhSLPI may provide a potential pharmaceutical target to limit endothelial-mediated I/R injury.

\section{Introduction}

Cardiac endothelial cells (ECs) serve an essential role in regulating blood perfusion, myocyte survival and contractile performance. The endothelium in the myocardial capillaries is in direct contact and communication with cardiomyocytes through a paracrine signalling pathway (1-3). Endothelial dysfunction due to ischaemia/reperfusion (I/R) has been reported to attenuate the flow or no-reflow phenomenon (1,4-6). In addition, the diapedesis of neutrophils from the endothelium into the interstitial myocardium is a hallmark of inflammation elicited by I/R (7). The activated endothelium and neutrophils release a variety of mediators to modulate cell injury, including proteases, oxygen-derived free radicals and pro-inflammatory cytokines (8). Endothelial dysfunction has been suggested as a trigger of the neutrophil amplification in I/R (8-10). In I/R injury, evidence has demonstrated that ECs are the first cell type to undergo apoptosis and to consequently migrate to neighbouring myocytes $(11,12)$. This would suggest that the endothelium is a more sensitive and soluble apoptogenic mediator from damaged endothelium that may diffuse into surrounding cardiac myocytes (11). In addition, the co-culture of ECs and cardiomyocytes under a hypoxia/reoxygenation $(H / R)$ injury was demonstrated to reduce the lactate dehydrogenase (LDH) activity and increase the nitric oxide production, suggesting that the endothelium may contribute to cardioprotection against an H/R injury (13). Therefore, it is hypothesised that maintaining endothelial function may be a potential strategy to minimise I/R injury.

Secretory leukocyte protease inhibitor (SLPI) is an inhibitor of serine protease regulating enzymatic activity and synthesis (14). Previously, it has been demonstrated that SLPI promotes early myocardial contraction, as well as inhibiting post-ischaemic inflammation in cardiac transplantation (15). Particularly during IR injury, increased protease production and intracellular $\mathrm{Ca}^{2+}$ overload promote the activation of $\mathrm{Ca}^{2+}$-dependent protease activity $(9,16)$. Thus, inhibition of the secreted protease activity may be an attractive target of SLPI, and may have a potential therapeutic effect in limiting cellular injury during I/R. However, the effect of SLPI in endothelial $\mathrm{I} / \mathrm{R}$ injury remains unexplored. 
Therefore, the aim of the present study was to determine the effect of recombinant human SLPI (rhSLPI) treatment against endothelial I/R injury in human umbilical vein ECs (HUVECs). The cell viability and expression of various mediators, such as lactase dehydrogenase (LDH) and reactive oxygen species (ROS), were assessed following rhSLPI treatment at different time points, including prior to ischaemia, during ischaemia and at the onset of reperfusion, in the cultured ECs subjected to I/R. Furthermore, the activation of cellular stresses, p38 mitogen-activated protein kinase (MAPK), protein kinase B (Akt), as well as the expression levels of apoptosis-regulating proteins, including B-cell lymphoma 2 (Bcl-2), Bcl-2-associated $\mathrm{X}$ protein (Bax) and cleaved caspase-3, were determined.

\section{Materials and methods}

Chemicals and reagents. rhSLPI was purchased from Sino Biological, Inc. (Beijing, China). Medium 200 (M-200-500), Low Serum Growth Supplement kit (S003K-LSGS) and trypsin-EDTA were purchased from Thermo Fisher Scientific, Inc. (Gibco; Waltham, MA, USA). The LDH liquid-UV assay kit was obtained from Human (Wiesbaden, Germany), and MTT was purchased from Ameresco, Inc. (Solon, OH, USA). Antibodies against total p38 (sc-728), phosphorylated-p38 (sc-17852-R), total Akt (sc-8312), phosphorylated-Akt (sc-293125), Bax (sc-6236), Bcl-2 (sc-783), and cleaved caspase 3 (sc-56053), $\beta$-actin (sc-130301), and the horseradish peroxidase-conjugated secondary antibody (sc-2004) were purchased from Santa Cruz Biotechnology, Inc. (Dallas, TX, USA). Other chemicals were purchased from Sigma-Aldrich (Merck KGaA, Darmstadt, Germany).

Cell culture. HUVECs were obtained from Thermo Fisher Scientific, Inc. (C01510C; Gibco) and were maintained in Medium 200 supplemented with the Low Serum Growth Supplement kit. Cells were maintained at $37^{\circ} \mathrm{C}$ at an atmosphere with $5 \% \quad \mathrm{CO}_{2}$ and $95 \% \mathrm{O}_{2}$ until they reached $80 \%$ confluence prior to performing any subsequent experiments.

Treatment protocol. HUVECs were cultured in 96-well plates (at 10,000 cells $/ \mathrm{cm}^{2}$ ) until $80 \%$ confluence was reached. Next, cells were treated with various concentrations $(0,1$, 10,100 and $1,000 \mathrm{ng} / \mathrm{ml}$ ) of rhSLPI at different time points, including $2 \mathrm{~h}$ prior to ischaemia (pretreatment group), at the onset of ischaemia until end of ischaemic period (during ischaemia group), and at the onset of reperfusion until the end of reperfusion period (reperfusion group). During these experiments, simulated ischaemia was performed for $40 \mathrm{~min}$, followed by $24 \mathrm{~h}$ of reperfusion. In the control group, cells were incubated with control buffer, during the simulated ischaemia. The different treatments are shown in more detail in Fig. 1.

Simulated $I / R(s I / R)$. In HUVECs, simulated ischaemia was induced by incubating the cells with a modified Krebs-Henseleit buffer (containing $137 \mathrm{mM} \mathrm{NaCl}, 3.8 \mathrm{mM}$ $\mathrm{KCl}, 0.49 \mathrm{mM} \mathrm{MgCl}_{2}, 0.9 \mathrm{mM} \mathrm{CaCl}_{2}$ and $4.0 \mathrm{mM} \mathrm{HEPES}$ ) supplemented with $20 \mathrm{mM}$ 2-deoxyglucose, $20 \mathrm{mM}$ sodium lactate and $1 \mathrm{mM}$ sodium dithionite at $\mathrm{pH} 6.5$, as previously described (17). The buffer used in the control group was composed of the Krebs-Henseleit buffer supplemented with $20 \mathrm{mM}$ D-glucose and $1 \mathrm{mM}$ sodium pyruvate. For optimizing the simulated ischemic duration to lead to cell death, cells were subjected to simulated ischaemia at $37^{\circ} \mathrm{C}$ in an atmosphere with $5 \% \mathrm{CO}_{2}$ for $40 \mathrm{~min}$, then the simulated ischaemic buffer was removed and replaced with complete medium for reperfusion and incubating at $37^{\circ} \mathrm{C}$ in $5 \% \mathrm{CO}_{2}$ for $24 \mathrm{~h}$. Cell viability was then measured via MTT assay. For the simulated ischemic duration that gave the strongest p38 MAPK activation, cells were exposed with simulated ischaemic buffer for different durations $(10,20,30,40,50$, and $60 \mathrm{~min})$. At each time point, the ischaemic buffer was removed and the cellular protein sample was collected by adding Laemmli sample buffer (62.5 mM Tris-HCl, pH 6.8, 25\% glycerol, 2\% SDS, $0.01 \%$ Bromophenol blue). Protein samples were kept at $-20^{\circ} \mathrm{C}$ prior to western blot analysis.

Measurement of cell viability. HUVECs were cultured as mentioned earlier and the cell viability was examined by an MTT assay. Briefly, subsequent to removal of the culture media, $0.5 \mathrm{mg} / \mathrm{ml}$ MTT reagent was added and incubated for $2 \mathrm{~h}$ at $37^{\circ} \mathrm{C}$. Following incubation, the excess MTT reagent was discarded and dimethyl sulfoxide (DMSO) was added to dissolve the formazan crystals. The sample was then centrifuged at $500 \mathrm{x} \mathrm{g}$ at $4{ }^{\circ} \mathrm{C}$ for $5 \mathrm{~min}$. The optical density was determined from supernatant fraction using a spectrophotometer at a wavelength of $490 \mathrm{~nm}$, using DMSO as the blank group. The relative percentage of cell viability was compared against the control group.

Measurement of cellular injury. The culture medium was collected from all experimental groups following completion of the sI/R protocol and kept at $-20^{\circ} \mathrm{C}$ untill released-LDH activity was determined using the $\mathrm{LDH}$ activity assay kit. Briefly, $10 \mu \mathrm{l}$ culture medium was mixed with $1,000 \mu \mathrm{l}$ reaction buffer and incubated at $37^{\circ} \mathrm{C}$ for $5 \mathrm{~min}$. Next, $250 \mu \mathrm{l}$ of substrate reagent was added, the solution was mixed and the absorbance was read after $1 \mathrm{~min}$ at a wavelength of $340 \mathrm{~nm}$. The mean absorbance changes per $\min (\Delta \mathrm{A} / \mathrm{min})$ were used to calculate the $\mathrm{LDH}$ activity with the following formula: $\mathrm{LDH}$ activity $(\mathrm{U} / \mathrm{l})=\Delta \mathrm{A} / \mathrm{min} \times 20,000$.

Determination of cellular ROS. For the determination of cellular ROS, $1 \times 10^{5}$ cells/ml HUVECs was cultured in a black 96 -well plate with complete medium at $37^{\circ} \mathrm{C}$ and $5 \% \mathrm{CO}_{2}$ until $80 \%$ confluence was reached. The culture media were removed and the cells were washed once with PBS prior to incubating with complete media containing $250 \mu \mathrm{M}$ 6-carboxy-2',7'-dichlorodihydrofluorescein diacetate (carboxy- $\mathrm{H}_{2}$ DCFDA) in a dark room for $30 \mathrm{~min}$ at $37^{\circ} \mathrm{C}$. The medium containing carboxy- $\mathrm{H}_{2}$ DCFDA was then removed and the cells were washed with PBS. For rhSLPI treatment, $200 \mu \mathrm{l}$ completed medium containing rhSLPI at the concentration $1,000 \mathrm{ng} / \mathrm{ml}$ was added and incubated for $1 \mathrm{~h}$ at $37^{\circ} \mathrm{C}$. Subsequently, $250 \mu \mathrm{M} \mathrm{H}_{2} \mathrm{O}_{2}$ was applied to the cells and incubated for $30 \mathrm{~min}$ at $37^{\circ} \mathrm{C}$. The intracellular ROS production was determined by measuring the fluorescence intensity using an EnSpire Multimode plate reader (PerkinElmer, Inc., Waltham, MA, USA) with a suitable set-up for detecting 


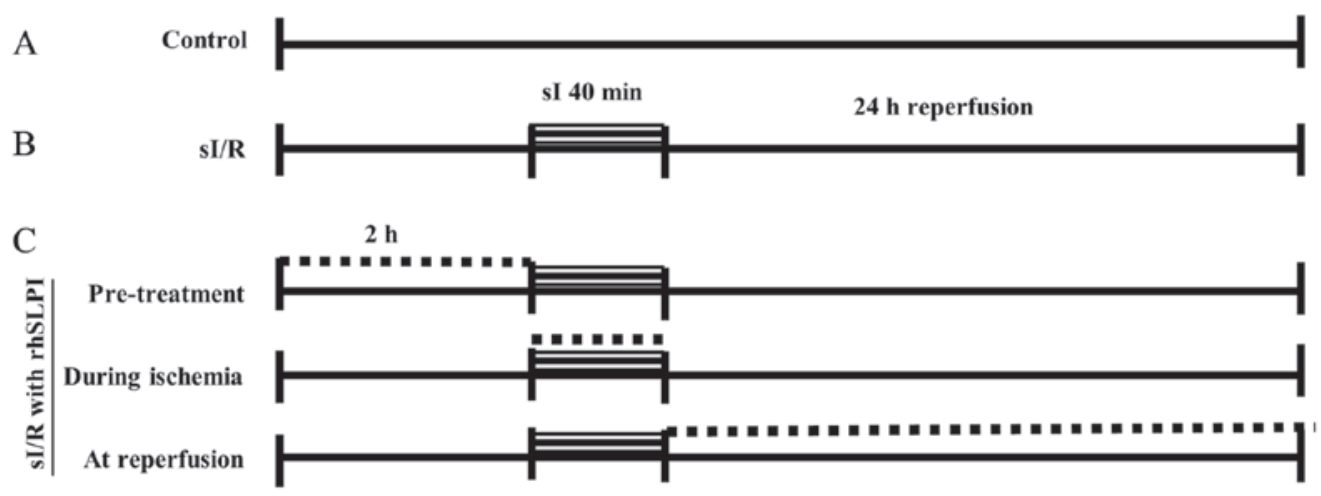

Figure 1. Diagram of the experimental protocol of sI/R performed in the present study. Human umbilical vein endothelial cells were divided into three experimental groups, as follows: (A) Control (normoxia); (B) sI/R (40-min ischaemia and 24-h reperfusion); (C) sI/R with rhSLPI treatment. rhSLPI at concentrations of $1,10,100$ or $1,000 \mathrm{ng} / \mathrm{ml}$ was added $2 \mathrm{~h}$ before ischaemia, during ischaemia (for $40 \mathrm{~min}$ ) or at the onset of reperfusion (for $24 \mathrm{~h}$ ). Dotted lines indicate the rhSLPI treatment duration. rhSLPI, recombinant human secretory leukocyte protease inhibitor; sI/R, simulated ischaemia/reperfusion; sI, simulated ischaemia.

the signal with an excitation wavelength of $498 \mathrm{~nm}$ and an emission wavelength of $522 \mathrm{~nm}$.

Measurement of MAPK activation by western blot analysis. The protein was extracted from the cells by adding for Laemmli sample buffer, $100 \mathrm{ng}$ protein of cell lysate was separated on a $12 \%$ SDS-polyacrylamide gel by electrophoresis and transferred to a polyvinylidene difluoride (PVDF) membrane. The PVDF membrane was then probed with the appropriate primary antibody by incubating at $4^{\circ} \mathrm{C}$ overnight with specific antibodies against total p38, phosphorylated-p38, total-Akt and phosphorylated-Akt at 1:1,000 dilution. In addition, the expression levels of apoptotic regulatory proteins were also determined by specific antibodies against Bax, Bcl-2 and cleaved caspase 3 (1:1,000). Membranes were subsequently washed using TBS/Tween-20 four times at room temperature and incubated with to horseradish peroxidase (HRP)-conjugated secondary antibody $(1: 2,000)$ for $1 \mathrm{~h}$ at room temperature. The antibody-antigen complexes were visualised by enhanced chemiluminescence using Luminata Crescendo Western HRP substrate (Merck KGaA) and detected using the Gel Doc XR+ system (Bio-Rad Laboratories, Inc., Hercules, CA, USA) Band densities were quantified using Image Lab software version 5.2.1 (Bio-Rad Laboratories, Inc.) and compared in order to provide information concerning the relative abundance of the protein of interest. Phosphorylated-p38 and -Akt were compared with total p38 and Akt, respectively, whereas Bax, Bcl-2 and cleaved caspase 3 were compared against $\beta$-actin as a reference protein.

Determination of cell morphology. HUVECs were grown on cell culture slides (SPL Life Sciences, Pocheon, Korea) at a concentration of $1 \times 10^{5}$ cells $/ \mathrm{ml}$ and cultured in complete medium at $37^{\circ} \mathrm{C}$ and $5 \% \mathrm{CO}_{2}$. Next, the cells were subjected to $40 \mathrm{~min}$ of simulated ischaemia followed by $24 \mathrm{~h}$ of reperfusion $(\mathrm{sI} / \mathrm{R})$ in the absence or presence of rhSLPI pretreatment or during ischaemia. Cells on the culture slides were then washed with PBS and fixed with a fixative agent (2\% formaldehyde and $0.05 \%$ glutaraldehyde) at room temperature for $30 \mathrm{~min}$. The cells were permeabilised with $0.5 \%$ Triton X-100 in PBS for $20 \mathrm{~min}$ and then stained with $50 \mu \mathrm{g} / \mathrm{ml}$ FITC-phalloidin conjugate (Sigma-Aldrich; Merck KGaA) for 40 min in a dark moist box for staining actin filaments. Subsequently, the cell culture slides were washed with PBS prior to nuclear staining with $0.01 \mu \mathrm{g} / \mathrm{ml}$ DAPI (Sigma-Aldrich; Merck KGaA) for $20 \mathrm{~min}$. The cell culture slides were then mounted by adding $20 \mu \mathrm{l}$ of $50 \%$ glycerol on a glass slide and sealing the edges with nail varnish. These samples were kept in a dark chamber until visualised under a fluorescence microscope (Carl Zeiss Jena GmbH, Jena, Germany).

Statistical analysis. All values are expressed as the mean \pm standard error of the mean. All comparisons were assessed for significance using analysis of variance, followed by the Tukey-Kramer test when appropriate. The statistical tests were performed using GraphPad Prism version 5 software (GraphPad Software, Inc., La Jolla, CA, USA). P<0.05 was considered to be an indicator of a statistically significant difference between the results.

\section{Results}

Optimisation of sI/R injury and examination of cytotoxicity of rhSLPI treatment. HUVECs were subjected to various periods of simulated ischaemia, followed by $24 \mathrm{~h}$ of reperfusion (sI/R). The results demonstrated that simulated ischaemia reduced the cell viability in a time-dependent manner (Fig. 2A). The sI/R at 40 min reduced the percentage of cell viability by $\sim 50 \%$, and thus this treatment duration was used in the sI/R protocol for determining the sI/R injury-induced cell death in subsequent experiments. In addition, HUVECs were treated with various concentrations of rhSLPI for $24 \mathrm{~h}$ prior to measuring cell viability. The results indicated that the cell viability was not affected by rhSLPI treatment and thus no cytotoxicity was observed (Fig. 2B).

Treatment of rhSLPI prior to or during simulated ischaemia protects against vascular EC death as a result of $S I / R$ injury. HUVECs were treated with various concentrations of rhSLPI in three different time points, including pretreatment, during the simulated ischaemia stage or at the onset 

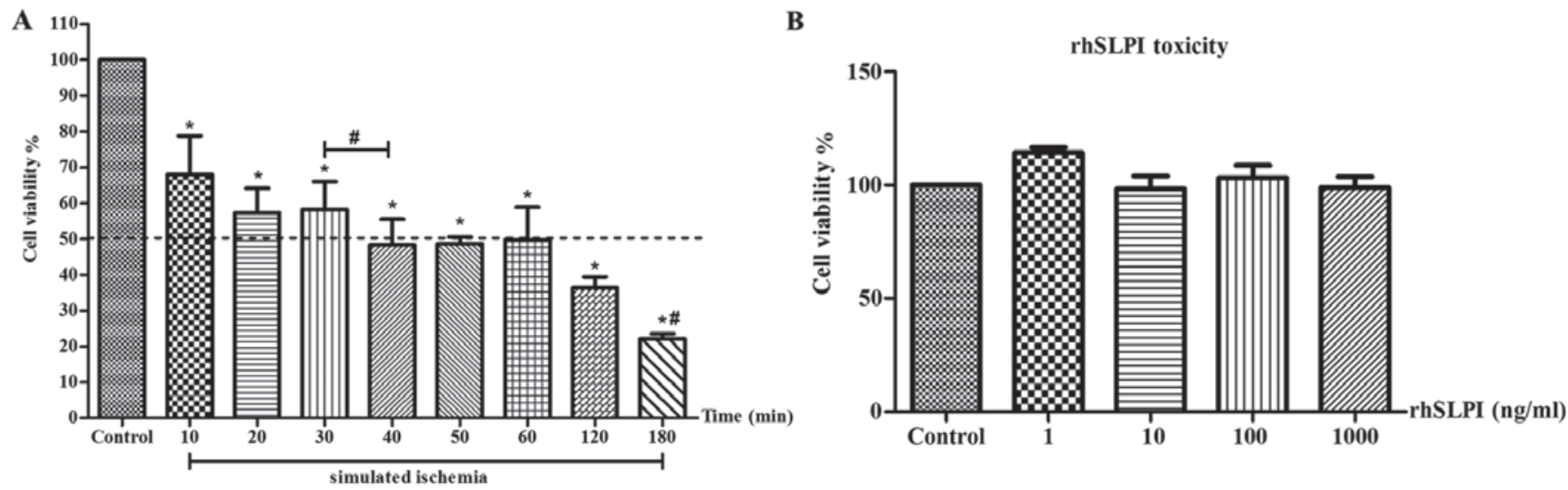

Figure 2. Optimised time for simulated ischaemia/reperfusion injury and investigation of rhSLPI cytotoxicity. (A) Viability of HUVECs subjected to simulated ischaemia for different durations $(10,20,30,40,50,60,120$ or $180 \mathrm{~min}$ ) followed by $24 \mathrm{~h}$ reperfusion. Cell viability was measured after reperfusion. (B) Viability of HUVECs after $24 \mathrm{~h}$ of rhSLPI treatment at the concentrations of 1, 10, 100 or 1,000 $\mathrm{ng} / \mathrm{ml}$ to determine the cell toxicity. The results are expressed as the mean \pm standard error of six experiments with independent cell preparations. " $\mathrm{P}<0.05$ vs. control group; ${ }^{\text {}} \mathrm{P}<0.05$ between groups. HUVECs, human umbilical vein endothelial cells; rhSLPI, recombinant human secretory leukocyte protease inhibitor.
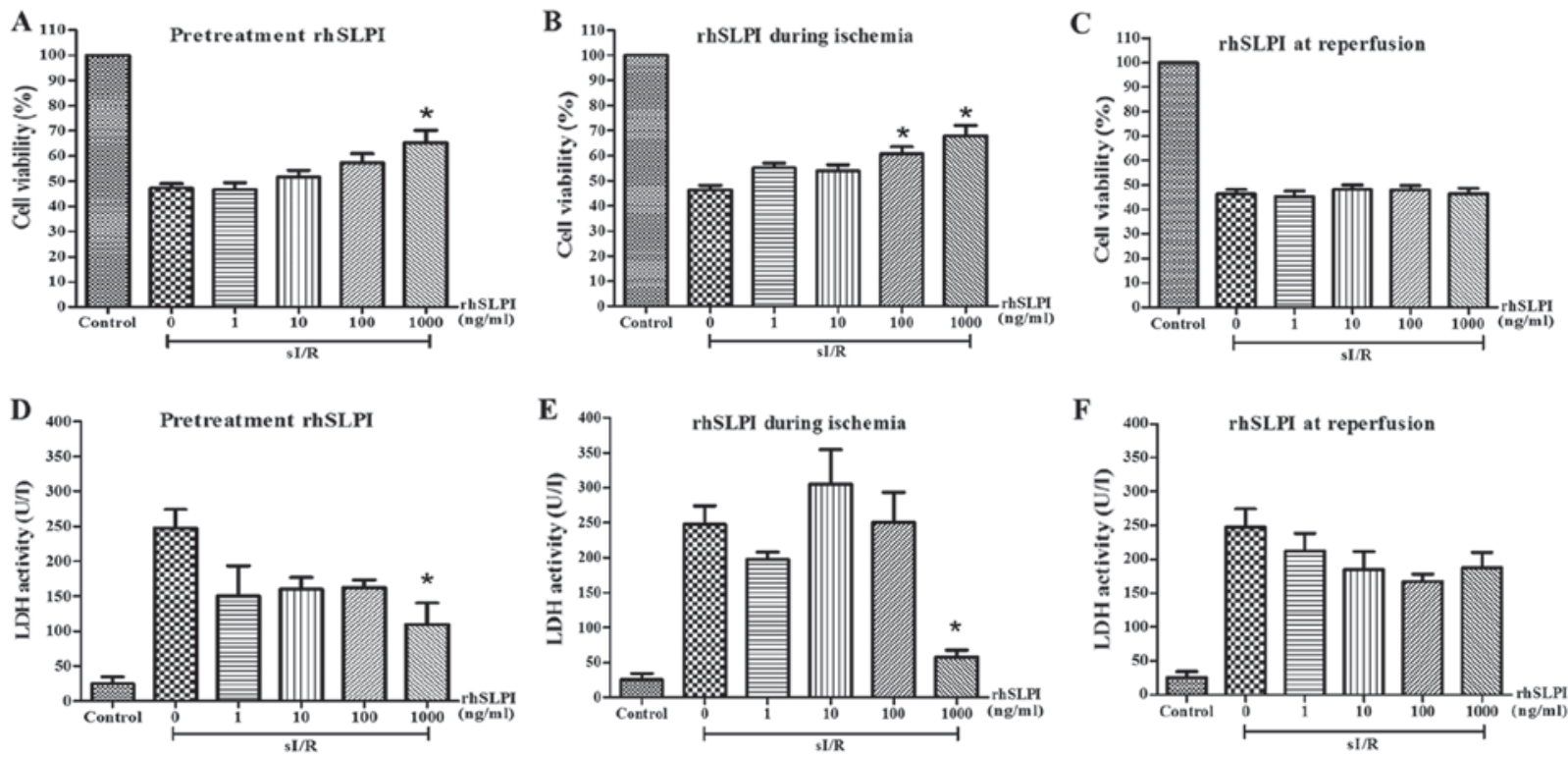

Figure 3. Effect of rhSLPI on endothelial cell viability. The cell viability was examined in HUVECs subjected to sI/R and treated with rhSLPI at the concentrations of $1,10,100$ or $1,000 \mathrm{ng} / \mathrm{ml}$ in the course of (A) $2 \mathrm{~h}$ before ischaemia, (B) for $40 \mathrm{~min}$ during ischaemia, or (C) at the onset of reperfusion for $24 \mathrm{~h}$. The medium of HUVECs treated with rhSLPI at different time points was collected for determining cellular injury by LDH activity assay when cells were treated (D) $2 \mathrm{~h}$ prior to sI/R, (E) during ischaemia and (F) at the onset of reperfusion. The results are expressed as the mean \pm standard error of 4-6 experiments with independent cell preparations. "P<0.05 vs. untreated sI/R group. HUVECs, human umbilical vein endothelial cells; rhSLPI, recombinant human secretory leukocyte protease inhibitor; sI/R, simulated ischaemia/reperfusion; LDH, lactate dehydrogenase.

of reperfusion. The results revealed that pretreatment with rhSLPI and treatment during the ischaemic period increased the cell viability and reduced the released LDH activity compared with the untreated sI/R group (Fig. 3). Among the pretreatment groups, the results indicated that pretreatment with rhSLPI at $1,000 \mathrm{ng} / \mathrm{ml}$ prior to sI/R significantly reduced the ischaemia-induced cell death when compared with the untreated group $(65.23 \pm 4.8$ vs. $47.16 \pm 1.8 \%$, respectively; $\mathrm{P}<0.05$; Fig. $3 \mathrm{~A}$ ). This concentration also significantly lowered the released LDH activity when compared with the untreated group $(104 \pm 30.3$ vs. $247.5 \pm 26.9$ U/I, respectively; $\mathrm{P}<0.05$; Fig. 3D). Furthermore, treatment with rhSLPI at 100 or $1,000 \mathrm{ng} / \mathrm{ml}$ during ischaemia significantly increased the cell viability $(67.88 \pm 4.2$ and $60.74 \pm 2.7 \%$ vs. $46.26 \pm 7.8 \%$, respectively; P<0.05; Fig. 3B). In terms of the released $\mathrm{LDH}$ activity, only rhSLPI at $1,000 \mathrm{ng} / \mathrm{ml}$ was able to markedly reduce the activity when compared with the untreated sI/R group (57.50 \pm 10.3 vs. $247.5 \pm 26.9 \mathrm{U} / \mathrm{I}$, respectively; $\mathrm{P}<0.05$; Fig. 3E). By contrast, all the concentrations of rhSLPI treatment at the onset of reperfusion did not demonstrate a significant difference in cell viability or the released LDH activity when compared with the untreated sI/R group (Fig. 3C and F).

Effect of rhSLPI on intracellular ROS production. To examine whether rhSLPI was able to reduce intracellular ROS production, an $\mathrm{H}_{2} \mathrm{O}_{2}$ challenge was performed. The results demonstrated that treatment using the $\mathrm{H}_{2} \mathrm{O}_{2}$ challenge resulted in a significant increase in intracellular ROS production by 


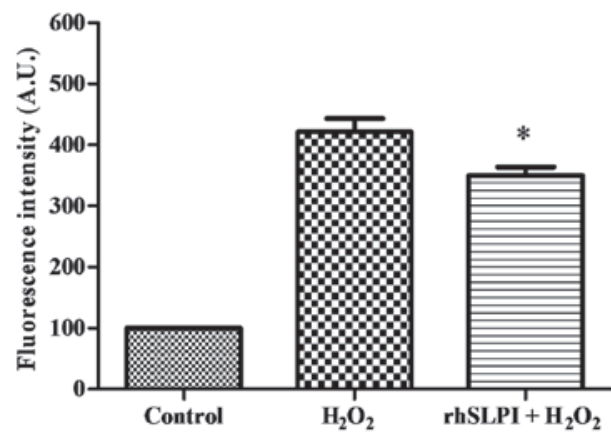

Figure 4. Effect of rhSLPI on the cellular ROS production. Human umbilical vein endothelial cells were incubated with control medium or pretreatmed with $1,000 \mathrm{ng} / \mathrm{ml} \mathrm{rhSLPI}$ prior to $\mathrm{H}_{2} \mathrm{O}_{2}$ addition for induction of cellular ROS production. The results are shown as the mean \pm standard error of four experiments with independent cell preparations. " $\mathrm{P}<0.05$ vs. $\mathrm{H}_{2} \mathrm{O}_{2}$ only group. rhSLPI, recombinant human secretory leukocyte protease inhibitor; ROS, reactive oxygen species.
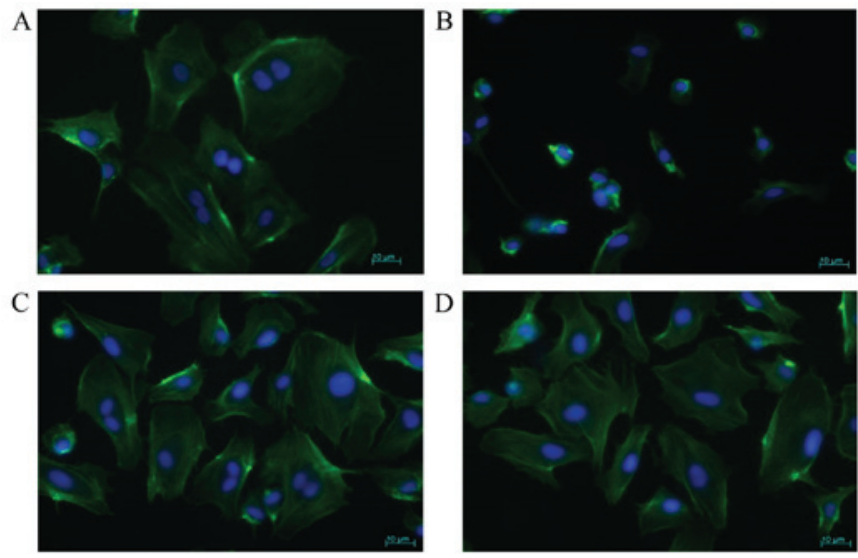

Figure 5. Effect of rhSLPI on cellular morphology. Human umbilical vein endothelial cells were treated with (A) control buffer, (B) sI/R alone, (C) pretreated with $1,000 \mathrm{ng} / \mathrm{ml} \mathrm{rhSLPI} 2 \mathrm{~h}$ prior to ischaemia followed by $\mathrm{sI} / \mathrm{R}$, or (D) treated with $1,000 \mathrm{ng} / \mathrm{ml} \mathrm{rhSLPI}$ for $2 \mathrm{~h}$ before subjected to simulated ischaemia or treatment of rhSLPI during ischaemia. The cells were subjected to actin cytoskeleton staining and examined under fluorescence microscopy in three experiments with independent cell preparations. rhSLPI recombinant human secretory leukocyte protease inhibitor; sI/R, simulated ischaemia/reperfusion

4-fold when compared with the control group (Fig. 4). However, pretreatment with 1,000 ng/ml rhSLPI was observed to significantly reduce the $\mathrm{H}_{2} \mathrm{O}_{2}$-induced oxidative stress by $\sim 17 \%$ compared with the $\mathrm{H}_{2} \mathrm{O}_{2}$ group (Fig. 4).

Effect of rhSLPI on cell morphology. To determine whether the treatment of protease inhibitor protected the cellular morphology and intracellular integrity, the HUVECs were treated with rhSLPI and exposed to sI/R. The results revealed that sI/R did not only cause cytoskeletal destruction, but also reduced the cell size and altered the cellular morphology when compared with the control cells (Fig. 5A and B). The disruption of the cytoskeleton was preserved in the ECs treated with $1,000 \mathrm{ng} / \mathrm{ml} \mathrm{rhSLPI}$ prior to ischaemia, as well as cells treated prior to ischaemia and stayed until the end of ischaemic period, as compared with simulated ischaemia alone (Fig. 5B-D).
Treatment of rhSLPI activates Akt phosphorylation and attenuates p38 MAPK activation. To determine cellular signalling in response to the rhSLPI treatment in HUVECs during sI/R injury, including the regulation of p38 MAPK and Akt, the cells were pretreated with $1,000 \mathrm{ng} / \mathrm{ml} \mathrm{rhSLPI}$ for $2 \mathrm{~h}$ before simulated ischaemia. Western blot assay was performed to analyse the phosphorylation of p38 MAPK and Akt. Initially, the simulated ischaemic period was optimised to determine the period required for marked activation of p38 MAPK. The results revealed that simulated ischaemia for $20 \mathrm{~min}$ led to a strong activation of p38 MAPK (Fig. 6A). Therefore, this treatment duration was then used to determine the effect of rhSLPI on signal activation. The results indicated that 20-min simulated ischaemia was able to strongly activate the p38 MAPK phosphorylation. Furthermore, pretreatment with $1,000 \mathrm{ng} / \mathrm{ml} \mathrm{rhSLPI}$ significantly reduced the p38 MAPK phosphorylation (Fig. 6B and C) and activated Akt phosphorylation (Fig. 6B and D), as compared with the simulated ischaemia alone. However, pretreatment with $1,000 \mathrm{ng} / \mathrm{ml}$ rhSLPI had no significant effect on the phosphorylation of $\mathrm{p} 38$ MAPK and Akt compared with controls.

Pretreatment of rhSLPI protects vascular ECs by attenuation of apoptotic regulatory protein activation. The EC regulatory pathway is a key determinant of cell death, particularly necrosis and apoptosis in I/R injury; thus, the effects of rhSLPI on the levels of apoptotic regulatory proteins, including Bax, Bcl-2 and caspase 3, were determined. The results of the present study revealed that pretreatment with $1,000 \mathrm{ng} / \mathrm{ml} \mathrm{rhSLPI}$ significantly reduced the level of Bax protein expression, the Bax:Bcl-2 ratio and the cleaved caspase-3 expression, with no marked changes observed in the levels of Bcl-2 expression (Fig. 7). This suggests that rhSLPI pretreatment leads to a reduction in apoptotic processes.

\section{Discussion}

ECs are sensitive to necrosis and apoptosis during I/R injury $(11,12)$. The ativated endothelium is an area of adhesion cascades involved in neutrophil extravasation $(7,12,18)$, aggravated inflammatory responses and accelerated microenvironment susceptibility to lethal injury (19). Thus, an attempt to preserve the EC integrity is an attractive therapeutic target in order to limit I/R injury.

To the best of our knowledge, the present study revealed for the first time that rhSLPI protects against in vitro I/R injury in ECs. The results also demonstrated the cytoprotective effect of rhSLPI treatment on ECs depending on the time point at which treatment was given. Administration of rhSLPI prior to or during ischaemia (for the entire period of ischaemia) was observed to potentially promote EC survival following sI/R injury, although treatment at a later stage upon the onset of reperfusion did not have an evident protective effect (Fig. 3). In addition, pre-incubation with rhSLPI manifested an inhibitory effect on $\mathrm{H}_{2} \mathrm{O}_{2}$-induced intracellular ROS production (Fig. 4), preserved the cytoskeletal organisation, reduced the p38 MAPK activation, activated the pro-survival kinase Akt, as well as reduced the expression levels of apoptotic regulatory proteins. 
A

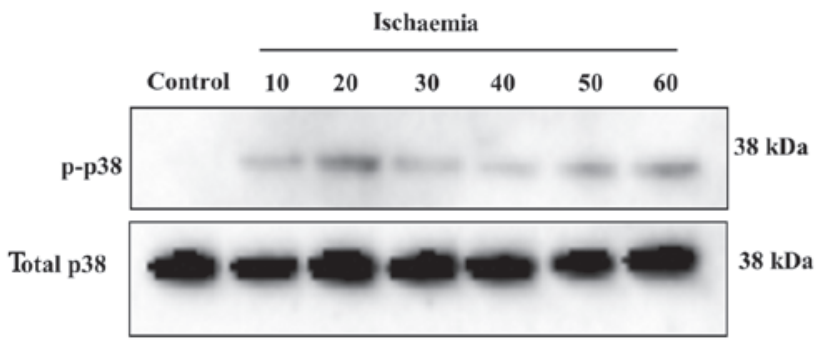

C

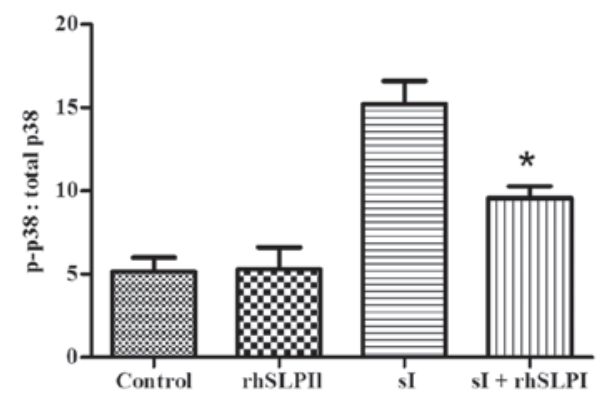

B

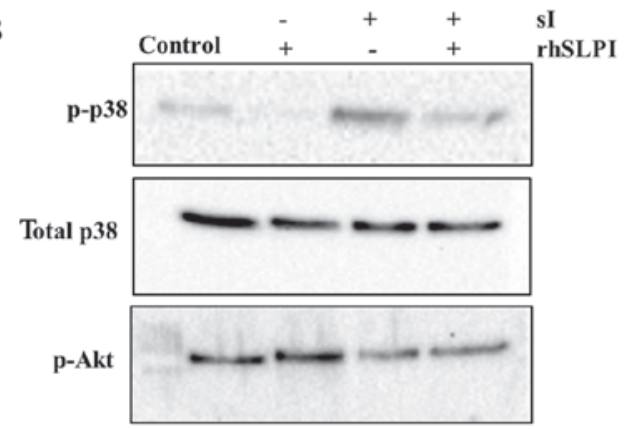

Total Akt

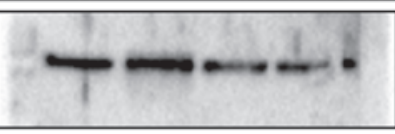

$\beta$-actin

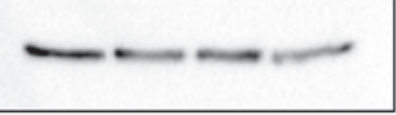

D

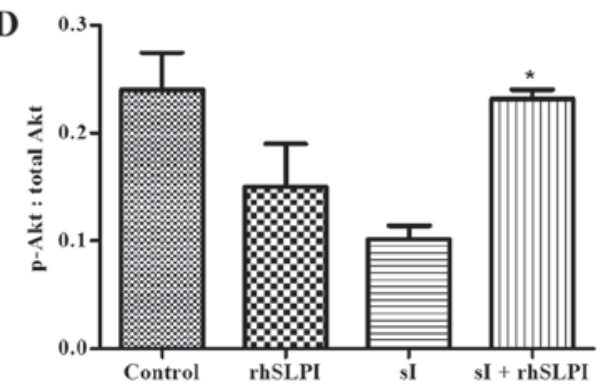

Figure 6. (A) Optimised ischaemia duration for MAPK activation in HUVECs. Cells were subjected to sI for 10, 20, 30, 40, 50 or 60 min, and the proteins expression of p38 MAPK was determined by western blot analysis in three experiments with independent cell preparations. (B) Effects of rhSLPI treatment on MAPK activation. HUVECs were subjected to control medium, pretreatment with 1,000 ng/ml rhSLPI without sI, $20 \mathrm{~min}$ of sI or 1,000 ng/ml rhSLPI pretreatment with 20-min sI. Endothelial proteins were prepared for western blot analysis to detect the expression levels of p38 and Akt. The quantified levels of (C) p38 and (D) Akt expressions were studied in 3-6 experiments with independent cell preparations. "P<0.05 vs. sI. HUVECs, human umbilical vein endothelial cells; rhSLPI, recombinant human secretory leukocyte protease inhibitor; sI/R, simulated ischaemia/reperfusion; sI, simulated ischaemia; Akt, protein kinase B; MAPK, mitogen-activated protein kinase.

A

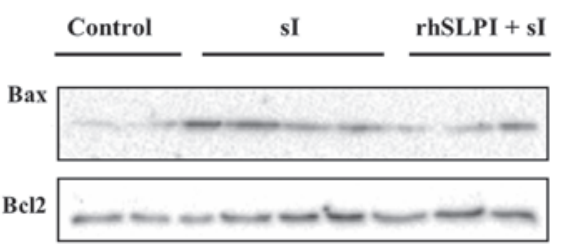

Caspase 3

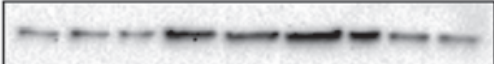

B
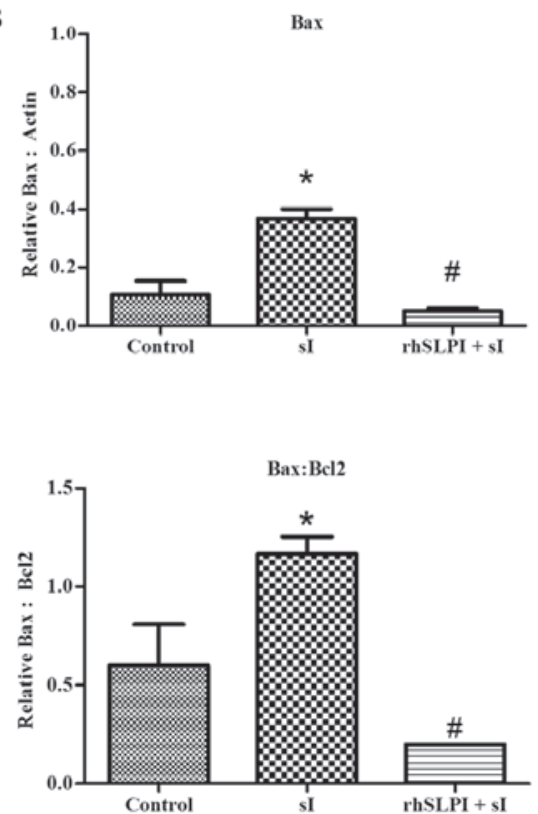
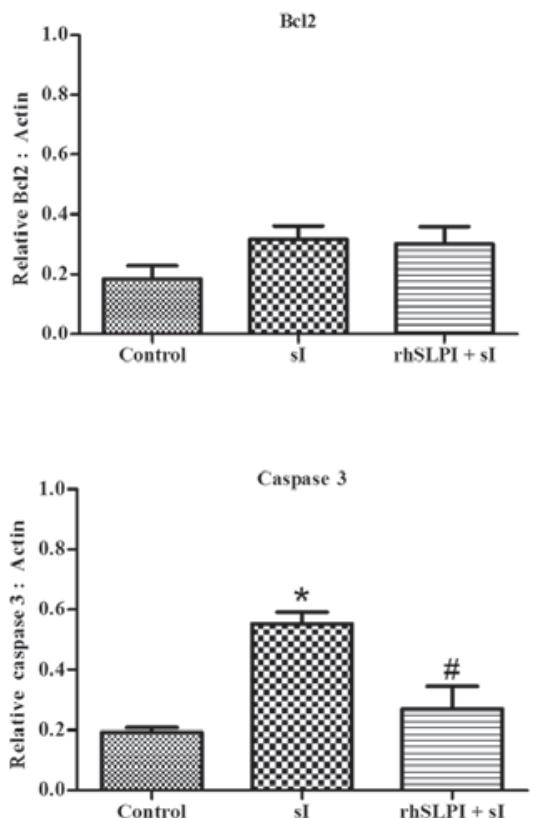

Figure 7. (A) Effects of rhSLPI on the activation of apoptotic regulatory proteins. HUVECs were subjected to control medium incubation, 20-min sI, or $1,000 \mathrm{ng} / \mathrm{ml}$ rhSLPI prior to ischaemia. Endothelial cell proteins were prepared for western blot analysis to detect the expression levels of Bax, Bcl-2 and cleaved caspase 3. (B) Quantified levels of Bax, Bcl-2, Bax: Bcl-2 ratio and cleaved caspase 3 were detected in 3-6 experiments with independent cell preparations. " $\mathrm{P}<0.05$ vs control group; ${ }^{\text {}} \mathrm{P}<0.05$ vs sI group. HUVECs, human umbilical vein endothelial cells; rhSLPI, recombinant human secretory leukocyte protease inhibitor; sI/R, simulated ischaemia/reperfusion; sI, simulated ischaemia; Bcl-2, B-cell lymphoma 2; Bax, Bcl-2-associated X protein. 
The present study also examined the significance of the period at which rhSLPI treatment should be provided in order to achieve protection against the sI/R-induced EC injury. Early rhSLPI administration was the most effective way to prevent deterioration of the injury, including intracellular ROS production and progression to apoptotic death. The most likely and effective intervention depends on the timing of drug administration; it should be given prior to reperfusion. However, the present study demonstrated the cytoprotective effect of rhSLPI and the significance of different time points of rhSLPI administration in vitro; consequently, in vivo experiments in further clinical studies are required to provide more clinically relevant findings. Therefore, the in vitro nature constitutes a limitation of the present study.

The endothelial protective ability of rhSLPI when added prior to the reperfusion period that was demonstrated in the present study is crucial for protection from cellular injury. When treatment of rhSLPI was applied prior to or during ischaemia, the protective effect remained over the reperfusion period. However, it remains unclear whether the actual cytoprotective mechanism of rhSLPI treatment is due to the inhibition of the protease activity by rhSLPI itself or the interaction of rhSLPI with other molecules. Previous studies reported that the phospholipid-binding protein Annexin II is a receptor of SLPI, while human ECs also express the Annexin II receptor $(20,21)$. However, whether the direct effect of rhSLPI on ECs occurs via interaction with phospholipid-binding protein Annexin II was not investigated in the present study and requires further investigation. In addition, the anti-protease activity of SLPI may possibly attenuate the protease mediated cell death and injury. However, the current study did not demonstrate whether rhSLPI protect EC death was due to the inhibition of protease activity. These associations remain unclear, constituting a further limitation of the present study.

The development of I/R injury involves a variety of mediators, including endothelial-derived ROS (8). The current findings demonstrated that treatment with rhSLPI attenuated the intracellular ROS production, suggesting the antioxidative property of SLPI in limiting the endogenous ROS generation during I/R, thus leading to cytoprotection against I/R injury. However, the potential suppressive effect of rhSLPI on endothelial ROS production in terms of the expression of specific effectors, including oxidative scavenging enzymes, superoxide dismutase, catalase and glutathione peroxidase, was not examined in the present study and needs to be considered for further investigation.

Signal transduction serves a key role in I/R injury and responses. Several protein kinases and cellular apoptotic regulatory proteins have been known to serve critical roles in the pathogenesis of I/R injury (7). The p38 MAPK, Bax, $\mathrm{Bcl}-2$, and caspase cascades are well defined as mediators of cellular apoptotic in I/R injury (22). In the current study, it was observed that rhSLPI attenuated p38 MAPK activation and pro-apoptotic Bax protein expression, as well as promoted pro-survival Akt protein activation under endothelial I/R injury (Figs. 6 and 7). These findings may explain the cytoprotective effect of rhSLPI in ECs against I/R injury. A limitation of the present study was that the results provided restricted mechanistic insights on the function of rhSLPI in ECs, since the protein levels of apoptotic factors $\mathrm{Bcl}-2$ and caspase- 3 may not provide sufficient information to confirm cellular apoptotic.
Therefore, an apoptosis-specific assay, such as TUNEL assay or Annexin V staining, should be conducted in future studies.

In conclusion, the present study is the first to report that rhSLPI protected against I/R-induced EC injury through the reduction of intracellular ROS production, attenuation of $\mathrm{p} 38$ MAPK, activation of pro-survival kinase Akt and reduced the levels of certain apoptotic factors. Furthermore, the data in the current study suggested that the therapeutic potential of rhSLPI in protecting vascular ECs from I/R injury provides a more significant clinical benefit when applied prior to reperfusion.

\section{Acknowledgements}

The present study was supported by the Naresuan University Endowment Fund (grant nos. R2558C085, R2559C007 and R2558B067), and the Royal Golden Jubilee PhD Program (grant no. PHD/0043/2555; joined funding between the Thailand Research Fund and Naresuan University) for PhD program scholarship.

\section{References}

1. Brutsaert DL: Cardiac endothelial-myocardial signaling: Its role in cardiac growth, contractile performance, and rhythmicity. Physiol Rev 83: 59-115, 2003.

2. Narmoneva DA, Vukmirovic R, Davis ME, Kamm RD and Lee RT: Endothelial cells promote cardiac myocyte survival and spatial reorganization: Implications for cardiac regeneration. Circulation 110: 962-968, 2004.

3. Hsieh PC, Davis ME, Lisowski LK and Lee RT: Endothelial-cardiomyocyte interactions in cardiac development and repair. Annu Rev Physiol 68: 51-66, 2006.

4. Schulz R, Kelm M and Heusch G: Nitric oxide in myocardial ischemia/reperfusion injury. Cardiovasc Res 61: 402-413, 2004.

5. Rezkalla SH and Kloner RA: No-reflow phenomenon. Circulation 105: 656-662, 2002.

6. Verma S, Fedak PW, Weisel RD, Butany J, Rao V, Maitland A, Li RK, Dhillon B and Yau TM: Fundamentals of reperfusion injury for the clinical cardiologist. Circulation 105: 2332-2336, 2002.

7. Kalogeris T, Baines CP, Krenz M and Korthuis RJ: Cell biology of ischemia/reperfusion injury. Int Rev Cell Mol Biol 298: 229-317, 2012.

8. Lefer AM and Lefer DJ: Pharmacology of the endothelium in ischemia-reperfusion and circulatory shock. Annu Rev Pharmacol Toxicol 33: 71-90, 1993.

9. Singhal AK, Symons JD, Boudina S, Jaishy B and Shiu YT: Role of endothelial cells in myocardial ischemia-reperfusion injury. Vasc Dis Prev 7: 1-14, 2010.

10. Eltzschig HK and Collard CD: Vascular ischaemia and reperfusion injury. Br Med Bull 70: 71-86, 2004.

11. Scarabelli T, Stephanou A, Rayment N, Pasini E, Comini L, Curello S, Ferrari R, Knight R and Latchman D: Apoptosis of endothelial cells precedes myocyte cell apoptosis in ischemia/reperfusion injury. Circulation 104: 253-256, 2001.

12. Chatauret N,Badet L,Barrou B and Hauet T: Ischemia-reperfusion: From cell biology to acute kidney injury. Prog Urol 24 (Suppl 1): S4-S12, 2014

13. Leucker TM, Ge ZD, Procknow J, Liu Y, Shi Y, Bienengraeber M, Warltier DC and Kersten JR: Impairment of endothelial-myocardial interaction increases the susceptibility of cardiomyocytes to ischemia/reperfusion injury. PLoS One 8: e70088, 2013.

14. Majchrzak-Gorecka M, Majewski P, Grygier B, Murzyn K and Cichy J: Secretory leukocyte protease inhibitor (SLPI), a multifunctional protein in the host defense response. Cytokine Growth Factor Rev 28: 79-93, 2016.

15. Schneeberger S, Hautz T, Wahl SM, Brandacher G, Sucher R, Steinmassl O, Steinmassl P, Wright CD, Obrist P, Werner ER, et al: The effect of secretory leukocyte protease inhibitor (SLPI) on ischemia/reperfusion injury in cardiac transplantation. Am J Transplant 8: 773-782, 2008. 
16. Powers SK, Murlasits Z, Wu M and Kavazis AN: Ischemia-reperfusion-induced cardiac injury: A brief review. Med Sci Sports Exerc 39: 1529-1536, 2007.

17. Jacquet S, Nishino Y, Kumphune S, Sicard P, Clark JE, Kobayashi KS, Flavell RA, Eickhoff J, Cotten M and Marber MS: The role of RIP2 in p38 MAPK activation in the stressed heart. J Biol Chem 283: 11964-11971, 2008.

18. Chen GY and Nuñez G: Sterile inflammation: Sensing and reacting to damage. Nat Rev Immunol 10: 826-837, 2010.

19. Kong Q, Dai L, Wang Y, Zhang X, Li C, Jiang S, Li Y, Ding Z and Liu L: HSPA12B attenuated acute myocardial ischemia/reperfusion injury via maintaining endothelial integrity in a PI3K/Akt/mTOR-dependent Mechanism. Sci Rep 6: 33636, 2016.
20. Ma G, Greenwell-Wild T, Lei K, Jin W, Swisher J, Hardegen N, Wild CT and Wahl SM: Secretory leukocyte protease inhibitor binds to annexin II, a cofactor for macrophage HIV-1 infection. J Exp Med 200: 1337-1346, 2004.

21. Cesarman GM, Guevara CA and Hajjar KA: An endothelial cell receptor for plasminogen/tissue plasminogen activator (t-PA). II. Annexin II-mediated enhancement of t-PA-dependent plasminogen activation. J Biol Chem 269: 21198-21203, 1994.

22. Kumphune S, Surinkaew S, Chattipakorn SC and Chattipakorn N: Inhibition of p38 MAPK activation protects cardiac mitochondria from ischemia/reperfusion injury. Pharm Biol 53: 1831-1841, 2015. 